\title{
Progress in the research on the antikaon-nucleon and nucleus interaction
}

\author{
Johann Marton*i \\ Stefan Meyer Institute for Subatomic Physics, Austrian Academy of Sciences \\ E-mail: Gohann.martondoeaw.ac.at
}

\begin{abstract}
The antikaon interaction on nucleons and nuclei in the low-energy regime is neither simple nor well understood. The research is concentrated on the one hand on x-ray spectroscopy of simple hadronic atoms with strangeness like kaonic hydrogen and helium isotopes to extract precision data on the strong interaction observables, on the other hand on the search of bound states like $\mathrm{K}^{-}$pp (production mechanism, binding energies, decay widths). In these research fields significant progress was made (e.g. SIDDHARTA at LNF). A European network LEANNIS within the European projects HadronPhysics 2 and HadronPhysics 3 is promoting the research on kaonic atoms and kaonic nuclei in Europe and outside Europe (e.g. Japan). This talk will give an overview of the progress and present status of experimental studies and will provide an outlook to future perspectives in this fascinating research field.
\end{abstract}

50th International Winter Meeting on Nuclear Physics

23-27 January 2012

Bormio, Italy

* Speaker.

${ }^{\dagger}$ for the LEANNIS network and SIDDHARTA 


\section{Introduction}

The research field of low-energy interactions of antikaons $\left(\mathrm{K}^{-}\right)$with nucleons and nuclei gained particular attention caused by the recent progress in theory and experiment. Experimental informations come from scattering experiments, kaonic atom radiative transition measurements and searches for kaonic clusters in various experiments. However, the antikaon interaction with the simplest systems like the the proton and the deuteron is still matter of intense research because of the opportunity of rather direct determination of the scattering lengths. The research field emerged about 4 decades ago when meson beams became available. A special and unique source of kaons is provided by the DAFNE $\Phi$ factory delivering monoenergetic $\mathrm{K}^{-}$at an energy of $\sim 16 \mathrm{MeV}$ from the $\Phi$ vector meson decay. In the case of kaonic hydrogen and deuterium the relevant observables are the shift $\varepsilon_{1 s}$ from the electromagnetic value of the $\mathrm{K}$ lines $(\mathrm{E}(\mathrm{K} \alpha)=6480 \mathrm{eV})$ and the broadening $\Gamma_{1 s}$ of the atomic ground state (1s) reflected by the K line width. The electromagnetic transition energy can be calculated with sufficient accuracy $(\sim 1 \mathrm{eV})$ by solving the Klein-Gordon equation and taking into account the various corrections like vacuum polarization and finite size effect. Since the strong interaction on the $n>1$ states is negligibly small and given the energy resolution of the spectroscopy system the measurement of the $\mathrm{np} \rightarrow 1 \mathrm{~s} x$-ray transitions gives direct access to these observables. The most severe complication for experiments is the rather small x-ray yield (in the case of $\mathrm{K}^{-} \mathrm{p}$ the yield is in the order of 1-3\%) demanding for high efficiency of the detector system and large background suppression. A puzzling situation was given after the first $\mathrm{x}$-ray experiments of kaonic hydrogen because the real part of the scattering length was found to have opposite sign in respect to the findings in $\mathrm{K}^{-}$-p scattering. This dilemma was settled by the experiments KEKPS E228 [四] and DEAR at DA $\Phi$ NE at LNF [व]. However, theory faced problems to extrapolate the sub-threshold behavior of the antikaon-nucleon interaction - especially due to the relatively small value of the $1 \mathrm{~s}$ state width $\Gamma_{1 s}$ found in the DEAR experiment. Therefore, a new series of $\mathrm{x}$-ray studies was conducted at DAFNE with the SIDDHARTA experiment employing silicon-drift detectors (SDDs) which provide timing information and consequently a huge suppression of kaonuncorrelated background events. New data on the low-energy strong interaction in kaonic hydrogen [B] and kaonic helium isotopes $\left(\mathrm{K}^{3} \mathrm{He}\right.$ [困] and $\mathrm{K}^{4} \mathrm{He}$ [[]]) were obtained which have strong impact in the theoretical description.

\section{New x-ray experiments}

In the SIDDHARTA experiment at the electron-positron collider DAFNE/LNF the nearly mono-energetic kaons from the $\Phi$ decay were used for the production of kaonic atoms like kaonic hydrogen and kaonic helium isotopes. An obstacle in the case of kaonic hydrogen is the relatively low $\mathrm{x}$-ray yield even in low-density gas $(\mathrm{Y} \sim 1-3 \%)$ which calls for high $\mathrm{K}$ - gas stopping and high detection efficiency of the $\mathrm{x}$-ray detector system. Therefore, the $\mathrm{x}$-ray transitions in these exotic atoms were measured with a ring-shaped array of Silicon Drift Detectors (SDDs) [目] providing a large solid angle, high intrinsic efficiency, excellent energy resolution (typical $\sim 150 \mathrm{eV}$ at 5.9 $\mathrm{keV}$ ) and timing information to overcome a limitation of the former DEAR experiment, i.e. the high asynchronous background. The use of the time correlation between the incoming kaon and 
the emitted x-ray suppresses the asynchronous background by orders of magnitude. With a pure hydrogen target filling the $\mathrm{K}$ transition series in kaonic hydrogen was measured (fig.1).

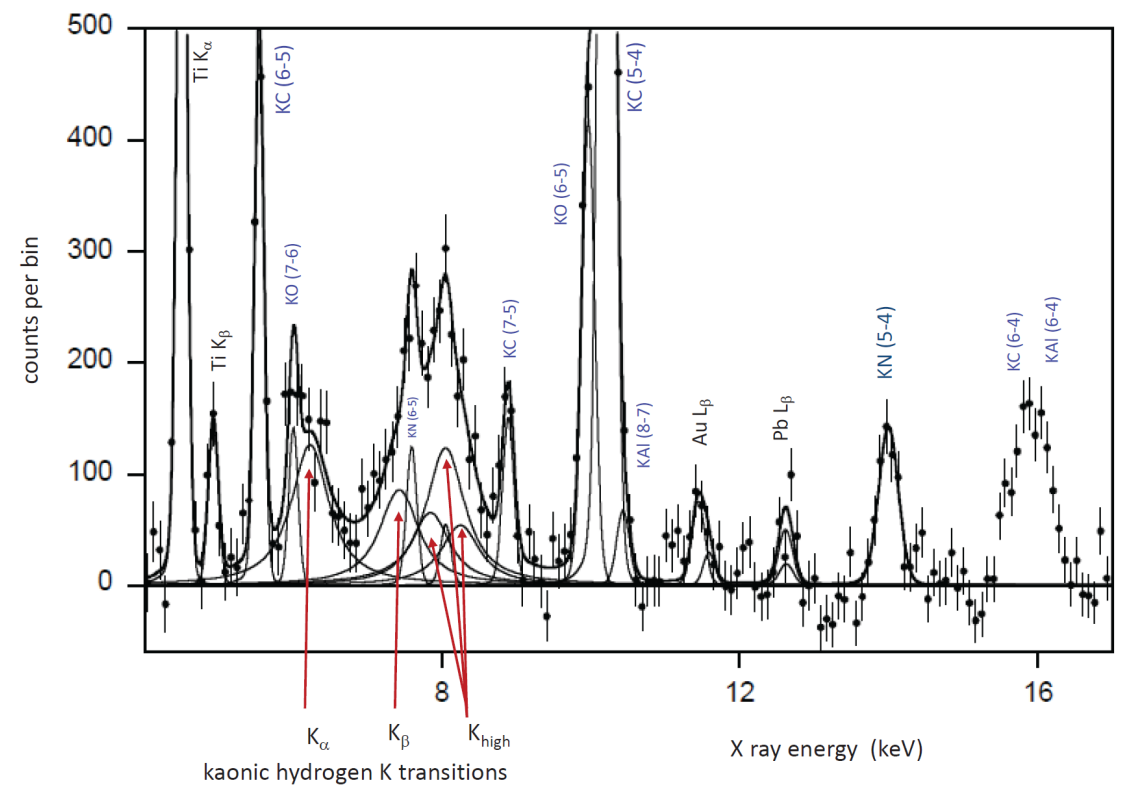

Figure 1: X-ray energy spectrum by SIDDHARTA using a pure cryogenic hydrogen gas target. The continuous background is subtracted. The peaks in the region of interest (solid line) are due to the $\mathrm{K}$ transitions $\mathrm{K}_{\alpha}, \mathrm{K}_{\beta}$ and the overlapping $\mathrm{K}_{h i g h}$. The unshifted position of $\mathrm{K}_{\alpha}\left(\varepsilon_{1 s}=0\right)$ is at $\sim 6.5 \mathrm{keV}$.

From the energy spectrum $\varepsilon_{1 s}$ and the broadening $\varepsilon_{1 s}$ due to strong interaction are extracted from the SIDDHARTA data which represent the most precise values [B]] up to now. Here $\varepsilon_{1 s}$ is defined as

$$
\varepsilon_{1 s}=E_{n p \rightarrow 1 s}^{\text {meas }}-E_{n p \rightarrow 1 s}^{e . m ., c a l c}
$$

where $\mathrm{E}_{n p \rightarrow 1 s}^{\text {meas }}$ stands for the measured energies of the $\mathrm{np} \rightarrow 1 \mathrm{~s}$ x-ray transitions and $\mathrm{E}_{n p \rightarrow 1 s}^{\text {e.m.,calc }}$ for the pure electromagnetic energies obtained from calculation.

$$
\begin{aligned}
& \varepsilon_{1 s}(K H)=-283 \pm 36(\text { stat }) \pm 6(\text { syst }) \mathrm{eV} \\
& \Gamma_{1 s}(K H)=541 \pm 89(\text { stat }) \pm 22(\text { syst }) \mathrm{eV} .
\end{aligned}
$$

For comparison the results of the latest experiments on kaonic hydrogen are plotted (see fig.2).

The precise determination of the strong interaction parameters is essential to determine the $\mathrm{K}^{-}$-p scattering length $\mathrm{a}_{p}$. For the extraction of $\mathrm{a}_{p}$ the Deser-Trueman formula [ $[\mathbb{}]$ was used in the past:

$$
\varepsilon_{1 s}+\frac{i}{2} \Gamma_{1 s}=2 \alpha^{3} \mu_{c}^{2} a_{p}
$$




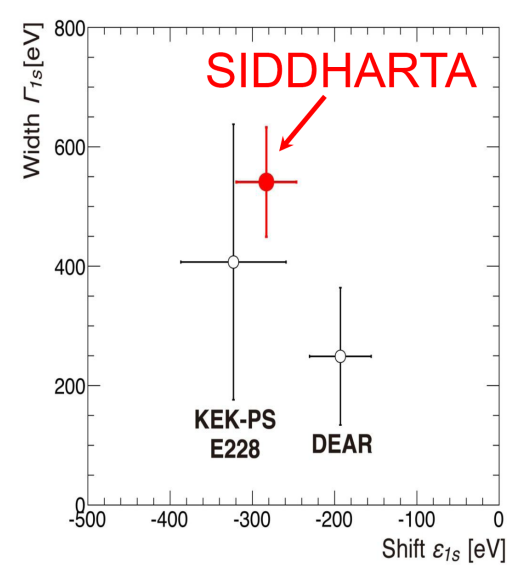

Figure 2: Hadronic shift and width from recent $x$-ray experiments on kaonic hydrogen. KEK-PS E228 [四], DEAR [] ] and SIDDHARTA [B]]. One can see that the SIDDHARTA delivered the most precise results up-to-now.

$\alpha$ is the fine structure constant and $\mu_{c}$ the reduced mass. It turned out that formula 2.4 has to be refined. An improved Deser-type formula take Coulomb corrections into account [8]

$$
\varepsilon_{1 s}+\frac{i}{2} \Gamma_{1 s}=2 \alpha^{3} \mu_{c}^{2} a_{p}\left(1-2 \alpha \mu_{c}(\ln \alpha-1) a_{p}\right)
$$

The result with formula 2.5 for $\mathrm{K}^{-} \mathrm{p}$ scattering amplitude $\mathrm{a}_{p}$ is given in 2.6. This result is perfectly consistent with the theory [Q] giving the best NLO result $\mathrm{a}_{p}=(-0.70+\mathrm{i} 0.89) \mathrm{fm}$.

$$
a_{p}=(-0.66+i 0.81) f m
$$

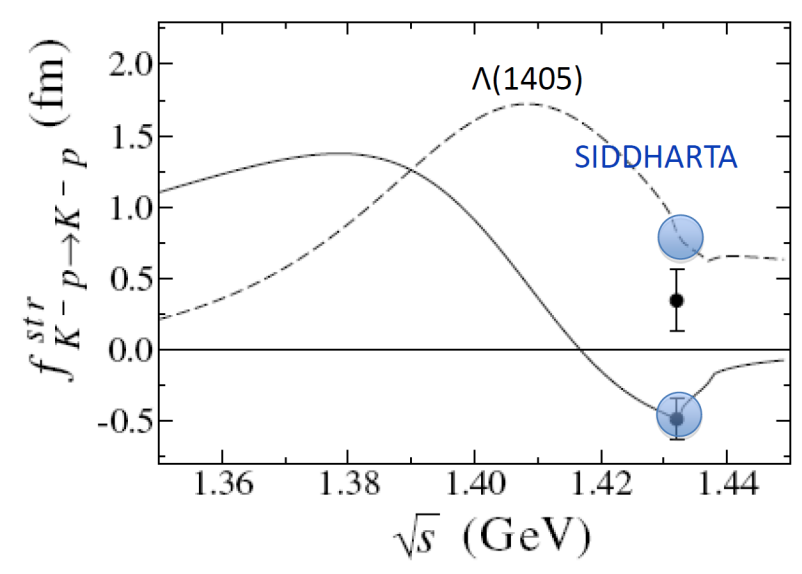

Figure 3: The scattering amplitude according to the ref.[ய] with the values for the real and imaginary part from SIDDHARTA indicated by circles. The black data points are the results from the DEAR experiment [[]]. 


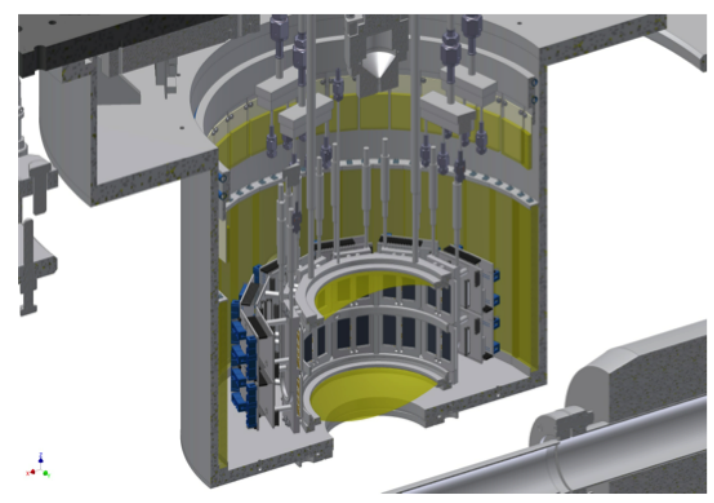

Figure 4: Cross section of the inner part of the SIDDHARTA2 experimental apparatus. The target cryogenic target cell is surrounded by an array of SDDs as x-ray detectors. The target and the SDD are cooled and mounted inside an insulation vacuum chamber.

\section{Outlook to SIDDHARTA2}

In spite of the success in getting the up-to-now most precise values of $\varepsilon_{1 s}$ and $\Gamma_{1 s}$ the case of kaonic deuterium is still open. SIDDHARTA performed a first exploratory measurement with a cryogenic gaseous deuterium filling of the target but due to the insufficient integrated luminosity and the obviously low x-ray yield the hadronic shift and width could not be extracted [ए]]. Nevertheless the measurement of the kaonic deuterium x-ray transitions is one of the most important tasks in the research field of low-energy QCD with strangeness. Thus the kaonic deuterium measurement has top priority for a dedicated experiment SIDDHARTA2 to be performed at DAFNE which is the follow up experiment after SIDDHARTA.

One of the main questions is the unknown yield for this reaction which depends strongly on the $2 \mathrm{p}$ state width. In a recent study [ए2] with two different models the yield for the $\mathrm{K}_{\alpha}$ transition was found as $0.27 \%$ for $2 \mathrm{p}$ state width $\Gamma_{2 p}=10.2 \mathrm{meV}$ and $1.9 \%$ for $\Gamma_{2 p}=2.7 \mathrm{meV}$ respectively. Many improvements are foreseen like a new target design and a new SDD arrangement. More cooling power will allow for higher gas densities and higher stopping efficiency. In order to suppress background an improved shielding employing also active veto detectors will be used.

There are some studies on the anticipated $\varepsilon_{1 s}$ and $\Gamma_{1 s}$ values in kaonic deuterium. Especially the width is an important input for the planning of SIDDHARTA2. According to recent calculations by the Bonn group [3] the $\mathrm{K}^{-} \mathrm{D}$ scattering length $\mathrm{a}_{d}$ is

$$
a_{d}=-1.46+i 1.08 f m
$$

and using the equation 2.5 with the kaonic deuterium numbers one gets for the kaonic deuterium strong interaction parameters

$$
\begin{aligned}
\varepsilon_{1 s}(K D) & =779 \mathrm{eV} \\
\Gamma_{1 s}^{d}(K D) & =650 \mathrm{eV}
\end{aligned}
$$

with estimated uncertainties of $\pm 170 \mathrm{eV}$ for the shift and $\pm 280 \mathrm{eV}$ for the width [[प]]. 


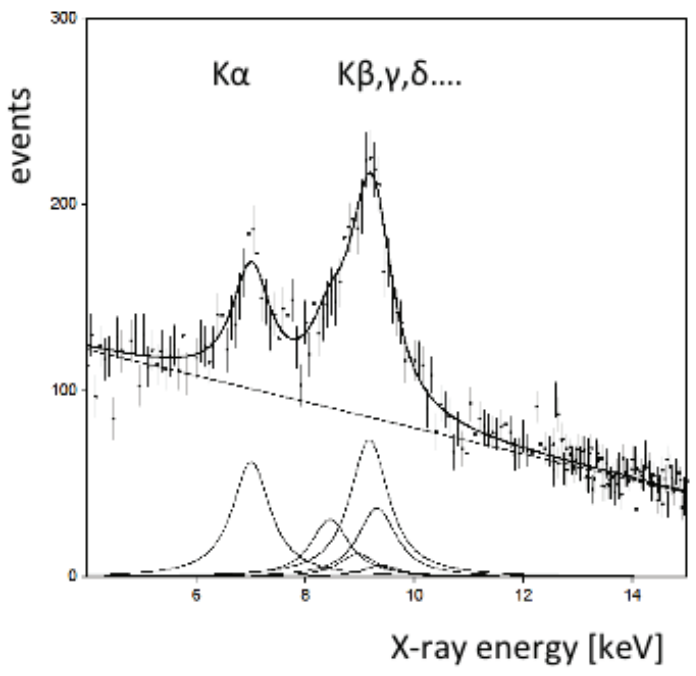

Figure 5: A Monte Carlo simulation of the kaonic deuterium $\mathrm{x}$-ray spectrum with the $\mathrm{K}_{\alpha}$ and hight $\mathrm{K}$ lines indicated. Here as input parameters $\varepsilon_{1 s}=-800 \mathrm{eV}$ and $\Gamma_{1 s}=800 \mathrm{eV}$ and an integrated luminosity of $800 \mathrm{pb}^{-1}$ were used.

Furthermore, the physics interest of SIDDHARTA2 covers also the strong interaction in other kaonic atoms with gas and solid targets and first attempts to measure the radiative $\mathrm{K}$ - capture on the proton [ㅍ]] which would give a direct access to $\Lambda(1405)$. Here again the yield of the $\gamma$ rays is unknown and probably small.

$$
K^{-} p \rightarrow \Lambda(1405)+\gamma
$$

\section{Searching kaonic nuclei}

Since the $\mathrm{K}^{-}$interaction is strongly attractive below threshold reflected also by the $\Lambda(1405)$ resonance the qustion was raised if it is possible to bind nucleons by $\mathrm{K}^{-}$attraction to get kaonic nuclei like the prototype system $\mathrm{K}^{-} \mathrm{pp}$ and have such bound states narrow widths to allow to find them in experiments [16]. The search for kaonic states of the type $\mathrm{K}^{-} \mathrm{pp}$ in various reactions is a hot topic also connected with kaonic atoms and the antikaon-nuclei interaction (for a review see [ए]]. A key subject is $\Lambda(1405)$ and its influence. According the Akaishi-Yamazaki [ए8] it could act as "doorway" state - essentially a bound $\mathrm{K}^{-} \mathrm{p}$ state - for the production of deeply bound kaonic nuclear clusters where the binding is given by the strong $\mathrm{K}^{-}$interaction. It was argued by theory that $\Lambda(1405)$ could have a more complicated structure (i.e. a 2-pole structure) due to the coupling. However this issue is also controversially discussed in theory [20]. Recently analyses of data from the DISTO Collaboration found indications for the production of $\mathrm{K}^{-} \mathrm{pp}$ in pp collisions [प्प]. An independent experiment with pp collisions at $3 \mathrm{GeV}$ was performed with the FOPI detector at GSI and these data are is in analysis at present. Also with FOPI a search for deeply bound kaonic 
states in heavy ion reactions was performed [R]]. There is ongoing work both in theory [[22] and experiment like AMADEUS at DAFNE [23, 24]].

\section{Summary and Outlook}

The SIDDHARTA experiment finished the kaonic hydrogen data analysis recently and obtained values of the low-energy strong interaction parameters with unprecedented accuracy. Thus improved constraints for theory are provided. The results triggered theoretical work in the testing ground of the $\mathrm{K}^{-}$-nucleon interaction at threshold. A consistent picture was achieved using effective field theory with coupled channels and putting together various sources of information like elastic scattering data. In spite of the big success of SIDDHARTA an extremely important information is still open - the case of kaonic deuterium has the highest priority for the follow-up experiment SIDDHARTA2.

\section{Acknowledgements}

This work was partly supported by the European Community Research Infrastructure Integrating Activity - Study of Strongly Interacting Matter- (HadronPhysics2, Grant Agreement No. 227431) under the Seventh Framework Programme of EU; Austrian Federal Ministry of Science and Research 650962/0001 VI/2/2009 and the networking activity LEANNIS within the European Community Research Infrastructure Integrating Activity HadronPhysics3 (Grant Agreement No. 283286).

\section{References}

[1] T. M. Ito, et al., Observation of Kaonic Hydrogen atom X rays Phys. Rev. C 58 (1998) 2366, M. Iwasaki et al., Observation of kaonic hydrogen $\mathrm{K}_{\alpha}$ X rays, Phys. Rev. Lett. 78 (1997) 3067.

[2] S. Bianco, et al., The DEAR Collaboration, La Rivista del Nuovo Cimento 22 (1999) 1; G. Beer et al., Measurement of the kaonic hydrogen spectrum, Phys. Rev. Lett. 94 (2005) 212302.

[3] M. Bazzi, et al., A new measurement of kaonic hydrogen X-rays, Physics Letters B 704 (2011) 113.

[4] M. Bazzi, et al., First measurement of kaonic helium-3 X-rays, Physics Letters B 697 (2011) 199.

[5] G. Beer, et al., Kaonic helium-4 X-ray measurement in SIDDHARTA, Physics Letters B 681 (2009) 310 .

[6] M. Bazzi, et al., Performance of silicon-drift detectors in kaonic atom X-ray measurements. Nuclear Instruments and Methods in Physics Research Section A 628 (2011) 264.

[7] S. Deser et al., Phys. Rev. 96 (1954) 774; T.L. Trueman, Nucl. Phys. 26 (1961) 57.

[8] U.-G. Meißner, U. Raha, A. Rusetsky, Eur. Phys. J. C 35 (2004) 349.

[9] Y. Ikeda, T. Hyodo and W. Weise, Improved constraints on chiral SU(3) dynamics from kaonic hydrogen, Physics Letters B 706 (2011) 63.

[10] B. Borasoy, R. Nißler and W. Weise, Phys. Rev. Lett. 94 (2005) 213401.

[11] M. Cargnelli et al., Proceedings of Science (STORI11) 042 and to be published. 
[12] M. Faber, et al., Energy-level displacement of excited np states of kaonic deuterium in a Faddeev-equation approach, Physical Review C 84 (2011) 064314.

[13] M. Döring und U.-G. Meißner, Phys. Lett. B 704 (2011) 663.

[14] M. Döring, private communication.

[15] S. Wycech, Kaonic atoms and $\Lambda(1405)$, Hyp. Int. (proceedings of EXA 2011) in print.

[16] E. Friedmann and A. Gal,In-medium nuclear interactions of low-energy hadrons, Phys. Rep. 452 (2007) 89.

[17] J. Zmeskal, From kaonic atoms to kaonic nuclei: A search for antikaon mediated bound nuclear systems, Prog. Part. Nucl. Sci. 61 (2008) 512.

[18] Y. Akaishi and T. Yamazaki, Phys. Rev. C 65 (2002) 044005.

[19] T. Yamazaki et al., Indication of a deeply bound and compact K- pp state formed in the pp- -> pLambdaK+ reaction at $2.85 \mathrm{GeV}$, Phys Rev Lett.104 (2010) 132502; P. Kienle et al., Role of the Lambda(1405) in the formation of the $\mathrm{X}-\mathrm{K}^{-} \mathrm{pp}$ deeply bound states revealed in the $\mathrm{pp} \rightarrow \mathrm{X}-\mathrm{K}^{+}$ reaction at $2.50 \mathrm{GeV}$ and $2.85 \mathrm{GeV}$, arXiv:1102.0482v1 [nucl-ex] (2011).

[20] E. Oset et al, Nucl. Phys. A 671 (2002), 481; J. Mares et al, Nucl. Phys. A 770 (2006), 84; N. Shevchenko et al, Phys. Rev. Lett. 98 (2007), 082301; W. Weise, H. Härtle, Nucl. Phys. A 804 (2008), 173; T. Hyodo, W. Weise, Phys. Rev. C 77 (2008) 035204.

[21] N. Herrmann et al., this Proceedings.

[22] M. Faber, et al., Molecule model for kaonic nuclear clusters $\bar{K}$ NN, International Journal of Modern Physics E 20 (2011) 1477.

[23] J. Marton, Low-energy antikaon nucleon interaction physics revisited, XLIX Int. Winter Meeting on Nuclear Physics, Proceedings of Science 063 (2011) 35.

[24] C. Curceanu (Petrascu), et al., Low-Energy Kaon-Nucleon/Nuclei Interaction Studies at DAFNE (SIDDHARTA and AMADEUS Experiments). Few-Body Systems 50 (2011) 447. 